\title{
COMPARATIVE STUDY OF PERCUTANEOUS NEEDLE ASPIRATION VERSUS CATHETER DRAINAGE IN TREATING HEPATIC ABSCESS
}

\author{
Brijendra Nigam¹, Manisha Srivastava2, Saket Nigam³, Chitra Chauhan ${ }^{4}$
}

1Associate Professor, Department of General Surgery, Rama Medical College Hospital and Research Centre, Kanpur, Uttar Pradesh, India.

2Associate Professor, Department of General Surgery, Rama Medical College Hospital and Research Centre, Kanpur, Uttar Pradesh, India.

${ }^{3}$ Associate Professor (Ex), Consultant, Department of Interventional Radiology, Regency Hospital, Kanpur, Uttar Pradesh, India. ${ }^{4}$ Assistant Professor, Department of Biostatistics, Rama Medical College, Kanpur, Uttar Pradesh, India.

\begin{abstract}
BACKGROUND
ABSTRACT

Pus Cavity in liver has been known cause of morbidity since the time of Hippocrates (400 BC). It is a common entity in the differential diagnosis of upper abdominal pain. The increasing incidence of Hepatic Abscesses reflects development and uses of more accurate diagnostic techniques like radioisotopes scan and subsequently ultrasonography and CT scan. Treatment of the liver abscess of any aetiology has been made simple and less morbid in the recent years. Percutaneous drainage of liver abscess and thereafter percutaneous pigtail drainage under ultrasonological guidance has been an important a dvancement in the treatment of liver abscesses as compared to conventional open drainage or conservative medicinal treatment. This study aims to investigate the effectiveness, morbidity and complication rates of Percutaneous Needle Aspiration in comparison to percutaneous continuous pig tail catheter drainage in the treatment of hepatic abscesses.

The objectives of this study were- 1 . to evaluate the clinical presentation, 2. to assess and compare the effectiveness of percutaneous continuous catheter drainage in comparison to needle aspiration in the treatment of liver abscesses.
\end{abstract}

\section{MATERIALS AND METHODS}

This prospective comparative study was conducted jointly at Rama Medical College and Regency Hospital, Kanpur from November 2014 to October 2018 on 100 patients of liver abscess, randomized equally into two groups, percutaneous needle aspiration group (PNA) and pigtail catheter drainage group (PCD). For percutaneous needle aspiration, lumbar puncture needle (size 18G to 21G) was used, while the size of pigtail catheter ( $8 \mathrm{f}$ to $12 \mathrm{f}$ ) was chosen according to thickness of pus assessed by ultrasonography. Both procedures were done under local anaesthesia with $2 \%$ Xylocaine. The effectiveness of either treatment was measured in terms of duration of hospital stay, days to achieve clinical improvement, $50 \%$ reduction in abscess cavity size and total/near total resolution of abscess cavity. Independent t-test was used to analyse these parameters. Only those patients having liver abscess/abscesses greater than $6 \mathrm{~cm}$ in at least one dimension, liquefied \& drainable were included in this study.

Statistical Analysis- Data was analysed by statistical software package SPSS version 21.0 (SPSS Inc. Chicago, IL, USA). The primary aetiology of abscess as per ultrasonological diagnosis recorded to compare the incidence of different causes of liver abscess in our area. The average (mean) value of duration of hospital stay, time taken for clinical signs of recovery (symptoms like pain, fever, laboratory parameters), mean duration of $50 \%$ reduction in size of abscess cavity and duration for near total or total resolution of abscess cavity in two groups as per ultrasonologic measurements of cavity dimensions have been compared. The average postoperative antibiotic treatment duration was noted. All data was analysed to compare the efficacy of two modes of treatment, using independent sample ' $t$ ' test. The ' $p$ ' value $<0.000$ is considered significant.

\section{RESULTS}

The study shows amoebic liver abscess is more common than pyogenic abscess in our area with hot humid climate. $50 \%$ reduction in volume of abscess cavity in catheter group was earlier as compared to those who underwent percutaneous needle aspiration. The patients in pigtail catheter drainage group showed earlier clinical improvement, and total or near total resolution of abscess cavity was found significantly earlier in per cutaneous catheter drainage technique. The procedure-related complications were found less in catheter group as compared with aspiration group. The overall clinical recovery was significantly earlier in catheter group than in needle aspiration group. Average duration of antibiotic treatment was significantly shorter in catheter group than in needle aspiration group.

\section{CONCLUSION}

Percutaneous pig tail catheter drainage is a better modality as compared to percutaneous needle aspiration especially in larger abscesses which are partially liquefied or abscesses with thick pus.

\section{KEY WORDS}

Liver Abscess, Pig Tail Catheter, Needle Aspiration.

HOW TO CITE THIS ARTICLE: Nigam B, Srivastava M, Nigam S, et al. Comparative study of percutaneous needle aspiration versus catheter drainage in treating hepatic abscess. J. Evolution Med. Dent. Sci. 2019;8(09):572-579, DOI: 10.14260/jemds/2019/128 
'Financial or Other Competing Interest': None.

Submission 03-01-2019, Peer Review 16-02-2019,

Acceptance 22-02-2019, Published 04-03-2019.

Corresponding Author:

Dr. Manisha Srivastava,

\#13 Hemant Vihar, Sector 3,

Barra 2, Kanpur-208027,

Uttar Pradesh, India.

E-mail: brijendramanisha@yahoo.com

DOI: $10.14260 /$ jemds $/ 2019 / 128$

\section{(c) $(1)(5)$}

\section{BACKGROUND}

Liver abscess has been recognized since Hippocrates (Circa 400 B.C.). He recognised different types of fluid in abscess cavity and speculated that prognosis of the patient was related to the type of fluid. ${ }^{1}$ Liver abscesses are most commonly due to pyogenic, amoebic or mixed infections. Although amoebic liver abscess occurs more commonly in men between 20 and 40 years of age, but can occur at any age. Approximately $60 \%$ are solitary and more frequently located in the right lobe of the liver, because most of the hepatic blood volume is in drained by portal blood flow mainly draining intestines, in right lobe of liver. When multiple abscesses are present, pyogenic or mixed is the most probable type. Patients usually present with a constant dull pain in the right upper quadrant of the abdomen which may be referred to the scapular region or the right shoulder with mild to moderate fever. Although amoebic liver abscess occurs more commonly on a worldwide basis and in tropics, the pyogenic liver abscess predominates in the United States. Earlier prime modality of treatment was medical and surgical intervention was needed in cases of refractory liver abscess (15\%) not responding to medical treatment, 2,3 and those which are secondarily infected $(20 \%)^{4}$ With laparotomy and drainage. ${ }^{5}$ However, operative drainage is associated with significant (10-47\%) morbidity and mortality. ${ }^{6}$ Recent developments in interventional radiology and frequent use of sonography and computerized tomography scanning of the abdomen, intensive care, advancements in antibiotic therapy have led to early diagnosis and easy and successful treatment outcomes of patients with liver abscess with reported success rates ranging from $70-100 \% .^{7-9}$ Percutaneous placement of an indwelling pig tail catheter (PDL) is the method most widely preferred to drain liver abscesses, ${ }^{10}$ than percutaneous needle aspiration of liver abscess. ${ }^{11,12}$ Although Needle aspiration is less expensive avoids problems related to catheter care and long-term hospital care. Multiple abscesses can be aspirated through different tracts in the same sitting. ${ }^{6}$ But needle aspiration has lower success rate than catheter drainage. ${ }^{7}$ Another problem with aspiration is that repeated needle aspirations (Average number per patient ranging from 1 to 3 may be required in a single patient over a short period of time from 5 to 14 days. This may be painful and unpleasant for the patients and hence may not be acceptable to them. ${ }^{8}$ To avoid these problems associated with needle aspiration, percutaneous pigtail catheter drainage is now used as the first method in the management of liver abscesses. The aim of this study is to confirm the advantage of catheter drainage which provides a continuous outlet to the pus and hence the problems of incomplete and repeated evacuations are not encountered, and total evacuation of abscess cavity occurs early leading to short period of morbidity with more success.

\section{MATERIALS AND METHODS}

This was a prospective randomized comparative study conducted in the departments of Surgery and Radiology, Rama Medical College, Kanpur and Department of Radiology, Regency Hospital, Kanpur. A total of 100 patients were included in the study, randomized into two groups; percutaneous needle aspiration group (PNA) and pigtail catheter drainage group (PCD). The study was conducted from November 2015 till October 2018. The group sample size has been calculated using the following formula $\mathrm{N} \geq(\mathrm{Z1}$ $\alpha / 2+\mathrm{Z} 1-\beta) 2(\sigma 12+\sigma 22) /(\mu 1-\mu 2) 2$, where Z1- $\alpha / 2$ is the Zvalue of type 1 error, maximum level assumed is $5 \%(\alpha=0.05)$ =1.96. $\mathrm{Z} 1-\beta$ is the $\mathrm{Z}$ value corresponding to power of the test, which has been fixed at minimum $80 \%$ for which the Value of Z1- $\beta=0.84$ Preliminary estimate for mean and standard deviation have been taken from the study 'Ann Gastroenterol. 2013; 26(4): 332-339.15 (The values for SD are 9.42 and 6.34. The values for means are 24.42 and 35.28 respectively). Using the results of this study, sample size came out to be more than $46(n>46)$ for each group, therefore sample size taken as 50 in both the groups (A \& B) for comparison, and a total of 100 patients taken into study.

The protocol was approved by the ethical review board of the institute. According to the principles of the declaration of Helsinki 1975, written, informed consent was obtained from all participants.

\section{Inclusion Criteria}

All the patients diagnosed to have liver abscess clinically confirmed by ultrasonography (USG) and /or CT scan were included in the study. The patients were selected from those attending the outpatient department and emergency department at both the hospitals. The age of patients varied from 16 to 60 years.

\section{Exclusion Criteria}

All abscess cavities smaller than $6 \mathrm{~cm}$ in their greatest dimension, prior intervention, ruptured liver abscess, uncertain diagnosis, concomitant biliary tract malignancy, and uncorrectable coagulopathy were excluded. A total of 112 patients were enrolled, and after assessment 10 patients were excluded due to prior PNA attempt, and 2 patients were excluded owing to a ruptured abscess.

All the patients evaluated for inclusion criteria, properly worked up and investigated with complete hemogram; liver function tests; prothrombin time; international normalized ratio (INR); activated partial thromboplastin time (APTT); amoebic serology; X-Ray Chest; abdominal USG with or without CT scan of the abdomen; and other investigations as per specific indications in different patients.

An informed consent was obtained from the participating patients and all the consenting patients were started on medical treatment as per our protocol.

Randomization was done using computer software, according to a standardized previously reported protocol. ${ }^{13} \mathrm{~A}$ set of random numbers generated were assigned to the two intervention groups and sealed numbered envelopes were made with the serial number mentioned on the outside and intervention mentioned inside by a non-participating 
individual. Once a participating subject gave valid consent the pre-determined intervention was carried out as follows: Patient were subjected to USG of the abdomen and the characteristics of the abscess cavity (ies) and pus were recorded.

The percutaneous procedures were carried out under local anaesthesia (2\% lignocaine) with IV analgesia and sedation if required, under continuous real-time USG guidance. A sample of pus was sent for Gram stain, culture, sensitivity and wet mount for Entamoeba histolytica trophozoites following both the procedures.

\section{Percutaneous Needle Aspiration (PNA) [Group A]}

Local anaesthesia was infiltrated at the proposed puncture site using a $23 \mathrm{G}$ needle. Under real-time USG guidance and using $18 \mathrm{G}$, BD spinal needle the abscess cavity was entered and pus was aspirated till no more pus could be aspirated further. A dressing was applied at the needle puncture site.

\section{Percutaneous Pig Tail Drainage (PCD) [Group B]}

Procedure carried out by placing 8-12 Fr pigtail catheter in the abscess cavity, under USG guidance, using the Seldinger technique, under local anaesthesia. Tract dilated with plastic dilators serially up to 8-12 Fr size. The appropriate Fr pigtail catheter then placed inside the abscess cavity and fixed to the skin using 2-0 Trulon suture. The catheter was attached to a collecting bag via the supplied connector. The size of pigtail catheter was chosen according to degree of liquefaction of pus inside abscess cavity assessed by USG.

All the patients empirically received injection Metronidazole 100ml (500 mg) IV BD. Injection Cefoperazone Sulbactam 1.5 g IV BD., Injection Amikacin 500 mg IV bd., The IV antibiotics and metronidazole were given for duration of 3 days and then Oral Tab. Cefuroxime 500mg BD, Tab Metronidazole $400 \mathrm{mg}$ t.i.d and Inj. Amikacin 500mg IM bd for total 5 days respectively. Chloroquine $500 \mathrm{mg}$ bid for 7 days followed by $300 \mathrm{mg}$ for 20 days (given in 2 divided doses). The empirical treatment was revised based on the culture and sensitivity report and duration of near total resolution of abscess cavity. Patients in whom pus culture was sterile continued on the same treatment.

\section{Evaluation of Clinical Response}

The pain, temperature and laboratory parameters e.g. Total Leukocyte count (TLC), liver function test (LFT), etc. were recorded every 2 nd day. In the patients undergoing PNA, USG was repeated after a gap of two days and aspiration repeated if the cavity size was still found to be greater than $5 \mathrm{~cm}$. The same procedure was repeated after a gap of another two days and aspiration repeated if needed.

The failure of clinical improvement in terms of fever, abdominal pain and tenderness and leukocytosis or failed reduction in size of the abscess cavity after a third attempt of aspiration was taken as failure of needle aspiration. These patients underwent PCD but were not added to the PCD group.

In patients who underwent PCD, besides recording the clinical and laboratory parameters of the patient every day, daily output of the catheter was measured, and the catheter was flushed with $20 \mathrm{cc}$ of normal saline (This volume was deducted from the total drainage). A decision to remove the pigtail catheter was made when the total drainage from the catheter decreased to less than $10 \mathrm{~mL} / 24 \mathrm{~h}$ for two consecutive days.

\section{Follow Up}

The patients were followed up weekly for a month, monthly for three months and at the end of six months, for clinical evaluation and USG assessment of abscess cavity until complete resolution of the abscesses was achieved. Data was collected and recorded in the printed proforma.

\section{Statistical Analysis}

Data was analysed by statistical software package SPSS version 21.0 (SPSS Inc. Chicago, IL, USA). The primary aetiology of abscess as per ultrasonological diagnosis recorded to compare the incidence of different causes liver abscess in our area. The average (Mean) value of duration of hospital stay, duration of appearance of clinical signs of recovery (symptoms like pain, fever, laboratory parameters), mean duration of $50 \%$ reduction in abscess cavity and duration for near total or total resolution of abscess cavity in two groups as per ultra-sonologic measurements of cavity dimensions have been compared. The average post-operative antibiotic treatment duration noted. All data analysed and compared to conclude the efficacy of two modes of treatment, using independent sample ' $\mathrm{t}$ ' test. The ' $\mathrm{p}$ ' value $<0.000$ is considered significant.

\section{RESULTS}

\section{Demographic Variation}

A total of 100 patients randomized into two groups (A \& B) of 50 each, were included in the study. The following observations were made:

The age of the patients varied from 16 years to 58 years with most of the patients falling within the age range from 20-40 years (60 patients). The second most common age group was 40-50 years (30 patients) and rest of patients was in extremes of age. There were 90 male and 10 female patients with liver abscess involved in the study. The male to female ratio was 10:1.

\section{Clinical Presentation}

Anorexia and pain in the right hypochondrium were commonest symptom, found in $97 \%$ \& $94 \%$ cases respectively. Moderate Prostration (92\%) and fever (90\%) were other frequently presenting symptoms. Approximately $58 \%$ weight loss, $56 \%$ has nausea or vomiting, and $48 \%$ night-sweats. Pain in the right shoulder region and cough were present in $38 \%$ and $37 \%$ of the patients respectively. 30 $\%$ has chills and rigor, $10 \%$ of the patients gave a history of diarrhoea prior to illness, and $8 \%$ has dyspnoea. In this study, hepatomegaly was found to be present in 72 of 100 patients $(72 \%)$ whereas pleural effusion was found in 8 of 100 patients (8\%). (Table 1, Figure 1)

\section{Lab Evaluation}

It was observed that 73 of 100 patients (73\%) had leukocytosis. Elevation of serum alkaline phosphatase was also observed in $70 \%$ of the patients. Amoebic serology 
positivity ( $>0.90$, EIA) was found in $65 \%$ of the patients Serum bilirubin more than $2.0 \mathrm{mg} / \mathrm{dl}$ was observed in $12 \%$ cases. Pus from all abscesses was sent for culture and sensitivity (Figure 2). Cultures were found to be positive in 28 of 100 (28\%) of the cases. The rest were sterile. Among the pus culture positive cases Escherichia coli was isolated most frequently i.e. 12 of 28 culture positive patients. It was closely followed by Klebsiella spp. which was isolated in 9 cases. Pseudomonas sp in 7 cases and Staphylococcus aureus were isolated in 7 patients. Amoebic liver abscesses were encountered more frequently (58\%) compared to pyogenic (23\%), amoebic abscesses with secondary bacterial infection (7\%) and abscesses of indeterminate aetiology (12\%) (Table 2 , Figure 3). The majority (about $85 \%$ ) of the abscesses were located in the right lobe of liver and rest $15 \%$ in the left lobe or in both lobes. About $80 \%$ of the cases studied were found to have solitary liver abscess cavity, whereas the rest of the patients had multiple abscesses $>3$ cavities. It was observed that the volume of the abscess cavities was mostly between $150-350 \mathrm{ml}$.

\section{Treatment Modality and Results}

All the 100 patients randomized in two groups. Group A (50 patients) underwent percutaneous aspiration and Group B (50 Patients) underwent pigtail Percutaneous drainage. The evidence-based response to treatment was recorded and analysed (Table 3). Pigtail percutaneous drainage was successful in all the 50 cases. On the other hand, imageguided needle aspiration (PNA) was successful only in 38 of 50 patients $(\mathrm{P}=0.006)$. Out of these (PNA) patients successfully treated, 21 patients required only one aspiration, 12 required two aspirations, and 5 required three aspirations. The 12 patients who did not show clinical improvement and/or decrease in cavity size despite 3 aspirations were taken as failures. The patients in PCD group showed earlier clinical improvement $(\mathrm{P}=0.036)$ and $50 \%$ decrease in abscess cavity volume $(\mathrm{P}=0.000)$ as compared to those who underwent PNA. Both group patients were kept under observation and for facilitating IV treatments for two days and thereafter patients of PCD group were discharged with catheter on. Both groups patients were called in OPD for regular follow up.

\begin{tabular}{|c|c|c|}
\hline \multicolumn{1}{|c|}{ Symptoms in Order of Decreasing Frequency } & \multicolumn{2}{c|}{ Percentage } \\
\hline Symptoms & No. of Patients & 97 \\
\hline Anorexia & 97 & 94 \\
\hline Weakness Upper Quadrant Pain & 94 & 92 \\
\hline Fever & 92 & 90 \\
\hline Weight Loss & 90 & 58 \\
\hline Nausea Vomiting & 58 & 56 \\
\hline Night Sweats & 56 & 48 \\
\hline Rt. Shoulder Pain & 48 & 38 \\
\hline Cough & 38 & 37 \\
\hline Chills and Rigor & 37 & 28 \\
\hline Diarrhoea & 28 & 10 \\
\hline Dyspnoea & 10 & 8 \\
\hline Hepatomegaly & 8 & 72 \\
\hline Pleural Effusion & 72 & 8 \\
\hline
\end{tabular}

\begin{tabular}{|c|c|c|c|c|}
\hline Treatment Group & Amoebic & Pyogenic & Mixed & Indeterminate \\
\hline PCD (Percutaneous Drainage Gp.) & 28 & 13 & 4 & 6 \\
\hline PNA (Percutaneous Needle Asp Gp.) & $30 \quad$ Table 2 & 10 & 6 \\
\hline
\end{tabular}

\begin{tabular}{|c|c|c|c|c|c|}
\hline \multicolumn{6}{|c|}{ Intervention and Results in Both Treatment Groups } \\
\hline \multirow[b]{2}{*}{ Parameters } & \multicolumn{2}{|c|}{ PCD Group } & \multicolumn{2}{|c|}{ PNA Group } & \multirow[b]{2}{*}{ 'p' Value } \\
\hline & $\begin{array}{c}\text { No. of } \\
\text { Patients }\end{array}$ & $\begin{array}{l}\text { Value Min-Max Mean + } \\
\text { SD }\end{array}$ & $\begin{array}{l}\text { No. of } \\
\text { Patients }\end{array}$ & $\begin{array}{l}\text { Value Min-Max } \\
\text { Mean + SD }\end{array}$ & \\
\hline Volume of Largest Cavity (C.C.) & 50 & $\begin{array}{c}96-580 \\
237 \pm 78.47 \\
\end{array}$ & 50 & $\begin{array}{c}122-510 \\
238 \pm 87.07\end{array}$ & 0.096 \\
\hline Procedural Success & 50 & $100 \%$ & 38 & $76 \%$ & 0.006 \\
\hline Hospital Stay (Days) & 50 & $3-6$ days $3.36 \pm 0.692$ & 50 & 1-7 days $3.5 \pm 1.69$ & 0.592 \\
\hline Clinical Improvement (Days) & 50 & $2-4$ days $2.98 \pm 0.68$ & 38 & 3-9 days $4.1 \pm 1.16$ & 0.036 \\
\hline $\begin{array}{c}\text { Duration Of } 50 \% \text { Reduction in Abscess Size } \\
\text { Cavity (Days) }\end{array}$ & 50 & 4-7 days $5.14 \pm 0.903$ & 50 & $4-12$ days $8.06 \pm 2.88$ & 0.000 \\
\hline $\begin{array}{c}\text { Duration of Near Total or Total Reduction in } \\
\text { Cavity (Weeks) }\end{array}$ & 50 & 7 -13 weeks $9.56 \pm 1.57$ & 50 & $\begin{array}{l}4-22 \text { weeks } \\
14.43 \pm 4.62\end{array}$ & 0.000 \\
\hline Duration of Drainage & 50 & 6 - 10 days $7.1 \pm 1.68$ & 50 & NA & NA \\
\hline \multicolumn{6}{|c|}{ Table 3} \\
\hline
\end{tabular}



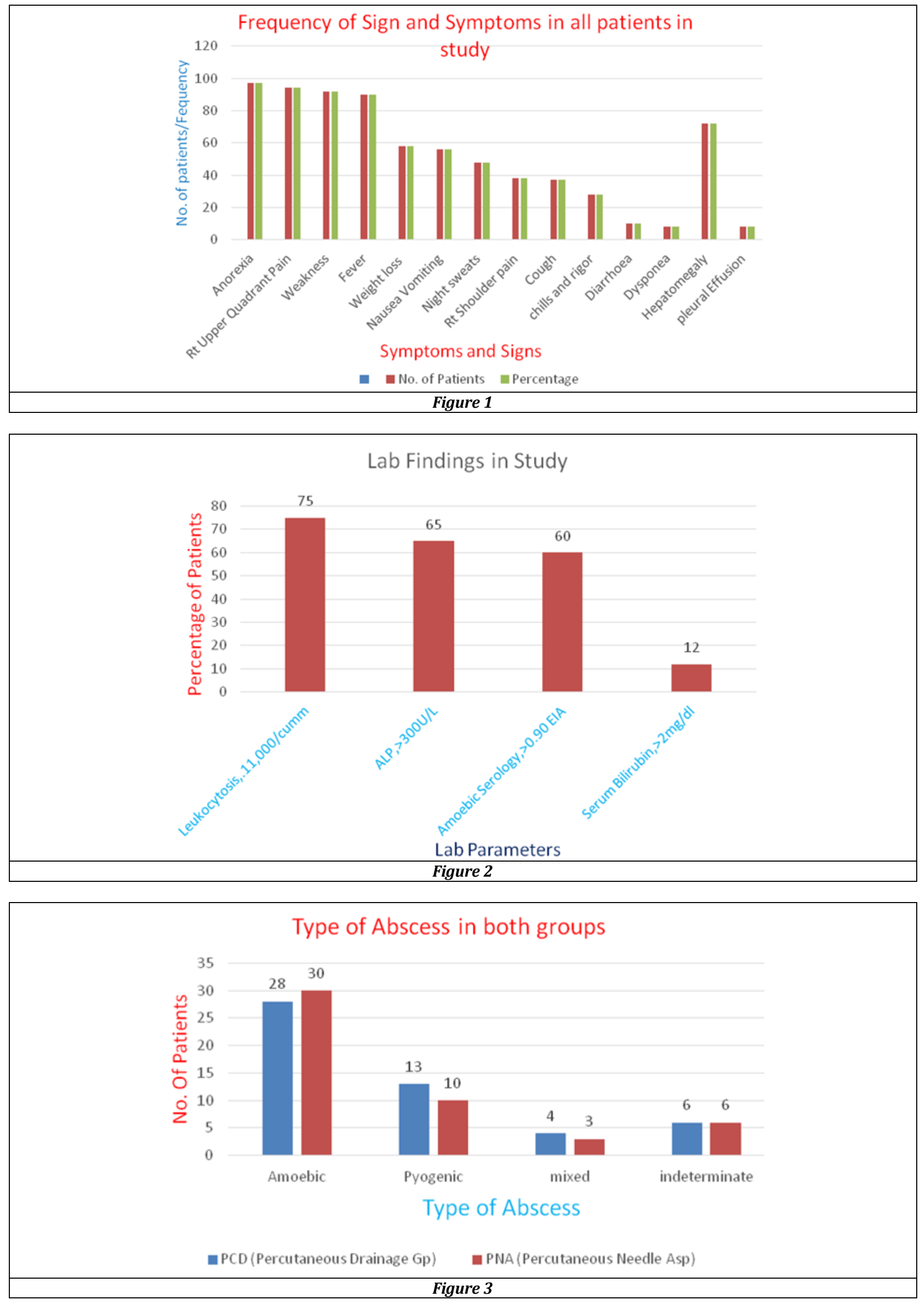


\section{DISCUSSION}

Liver abscess is a major tropical disease of the gastrointestinal system.14,15 The liver abscess is mainly classified into amoebic and pyogenic. In our study we found the male to female ratio to be 10: 1 . The most frequently affected age group was in the third and fourth decade.

The clinical presentation of the patients studied in our series was similar to the descriptions in previous reports. 16 The presenting clinical symptoms and signs of liver abscess in our study were fever (90\%), right upper quadrant pain and tenderness (94\%) and hepatomegaly (72\%). These clinical manifestations are similar to those described in previous studies. ${ }^{17,18}$

In our study, $85 \%$ of the abscesses were located in the right lobe of liver while $15 \%$ were located in either left lobe or in both lobes, similar to previous studies 16,19 . $80 \%$ of our patients had solitary abscesses, similar to a previous report. ${ }^{20}$ We encountered multiple liver abscesses in $20 \%$ of the patients, similar to the $20-25 \%$ incidence of multiple liver abscesses reported by Sharma et al. ${ }^{21}$

The type of abscess was determined on the basis of amoebic serology and pus culture reports. ${ }^{22}$ In our study we found $58 \%$ of the abscesses to be amoebic in aetiology, $23 \%$ to be pyogenic, $12 \%$ to be indeterminate and $7 \%$ to be amoebic with secondary bacterial infection (or mixed liver abscess). Khan et al in their series reported $68 \%$ amoebic, $21 \%$ pyogenic, $8 \%$ indeterminate, and 3\% MLA. The use of serological testing for diagnosis of amoebic liver abscesses can occasionally lead to either false negative results early in the course of the disease, due to delay in rise of antibody titer, or to false positives due to background subclinical amoebic infections. Consideration of high titers for diagnosis may help exclude these false positives. ${ }^{23}$

The pus cultures were negative in 76 of 100 patients. Aerobic cultures were declared negative after $48 \mathrm{~h}$ of incubation. There were 12 patients $(12 \%)$ in whom the amoebic serology as well as pus cultures were negative. As several of our patients prior to reporting to our hospital had been given antibiotics as well as anti-amoebic drugs, this might explain the finding of $12 \%$ cases with indeterminate aetiology. Similar experience has been reported by other researchers as well.

Pus culture was found positive in $24 \%$ cases. The Escherichia coli (44\%) was most frequently isolated bacteria on pus culture clos followed by Klebsiella species (33\%). These finding were similar as reported in other studies. ${ }^{9,24,25,26}$

100 patients underwent image guided intervention of uncomplicated liver abscess and we obtained good results. There was no mortality or any major complication requiring any treatment which also conforms with previous documented studies about the safety and excellent results of image guided percutaneous procedures.20,27,28,29 Open surgical drainage is usually reserved for patients who have failed percutaneous drainage, those who require surgery for management of underlying problems, and some patients with multiple macroscopic abscesses. ${ }^{30}$ It can be said that in recent years image-guided percutaneous treatment (Needle aspiration or catheter drainage) has replaced surgical intervention as the primary treatment for liver abscess. ${ }^{7-10,31,32}$
In several other studies good results were obtained with PNA along with systemic antibiotics. Giorgio et al performed on an average 2.2 aspirations in 115 patients and reported resolution of symptoms and hepatic lesions in $98 \%$ of the patients. In our study we treated 50 patients with PNA along with systemic antibiotics. Of these 50 patients, 38 were successfully treated with 21 requiring only one aspiration, 12 requiring a second aspiration and 5 patients requiring a third aspiration as well. 12 patients $\mathrm{s}$ failed to improve clinically and did not show significant decrease in abscess cavity even after 3 aspirations. Thus, 38 patients (76\%) who were successfully treated with aspiration required an average of 1.6 aspirations. Rajak et al $^{33}$ reported a success rate of $60 \%$ with needle aspiration. However, in their study only two attempts of aspiration were made and failure to attain clinical, haematological and radiological improvement was taken as failure of therapy.

The mean duration of time taken for clinical improvement was $4.1 \pm 1.16$ days in this modality of treatment as compared to $2.98 \pm 0.68$ in PCD group. The time required for $50 \%$ reduction in the cavity size was significantly less in the PCD compared to PNA group $(5.14 \pm 0.903)$ days and $8.06 \pm$ 2.88 days respectively, $\mathrm{P}=0.000$ ). However, time required for total or near-total resolution of the cavity in the two groups $(\mathrm{PCD}=9.56 \pm 1.57$ weeks, $\mathrm{PNA}=14.43 \pm 4.62$ weeks, $\mathrm{P}=0.000)$ was found to be significant.

Until recent times PNA was preferred over PCD because it is less invasive and less expensive; avoids problems related to catheter care; and multiple abscess cavities can be aspirated easier in the same setting. However, in our study we had a success rate which was significantly lower than with catheter drainage $(76 \%$ versus $100 \%, \mathrm{P}=0.006)$. There are some problems with catheter drainage like nuisance to the patient, pain, cellulites at the insertion site and sometimes catheter dislodgement.

The success rate in our study after single aspiration was $42 \%$ after second aspiration $66 \%$ and after third aspiration it was $76 \%$. The success rate of PNA in the literature varies from $79-100 \% \cdot 10,35$ Although, needle aspiration is a much simpler procedure when compared to catheter drainage repeated procedures are quite unpleasant and traumatic for the patients and may not be acceptable to many. Even after repeated aspirations the success rate was far from being $100 \%$. Therefore, those patients who failed after a third aspiration attempt were offered catheter drainage.

The average size of abscess in our study was $237 \pm 78.47$ $\mathrm{mL}$ and $238 \pm 87.07 \mathrm{~mL}$ for the PCD and PNA group respectively, comparable to the study reported by Rajak et al ( $335 \mathrm{~mL}$ and $221 \mathrm{~mL}$ respectively). The success rate achieved by Rajak et al was $60 \%$, comparable to the success rate after the second aspiration in our study, i.e. 66\%. Subsequent aspirations seem to improve the success rate of therapy.

In our study we found that larger abscesses are more difficult to evacuate completely in one attempt, necessitating subsequent aspirations, in contrast to some of the earlier reports that show that the initial size of the abscess cavity did not affect the ultimate outcome.

Other reasons for failure of needle aspiration is the inability to completely evacuate the thick viscous pus that may be present in some of the abscesses and rapid reaccumulation of pus in the abscess. ${ }^{34}$ 
Placement of an indwelling drainage catheter addresses all three of these issues as it provides continuous drainage, drains thick pus because of wider caliber catheter, and prevents re-accumulation. This explains the higher success rates $(100 \%)$ observed in our study and several previous studies.11,33,31,35

The reasons for failure of PCD as reported in some of the earlier series have been attributed to either thick pus not amenable to percutaneous drainage (wider bore catheter used in our series) or premature removal of drainage catheter. No recurrence occurred in any of our cases during the follow up period. However, both treatment modalities resulted in rapid clinical relief with most patients showing resolution of signs and symptoms within the first 4 days of the procedure.

It can be concluded that the abscess cavities showed faster collapse during the initial period in the PCD group and it did have significant advantage as far as total or near-total resolution of cavity is concerned. Thus, our study differs in this observation from study of previous investigators.

Complications such as haemorrhage, pleural effusion/empyema, persistent bile drainage, catheter displacement, sepsis etc., have been reported with both PNA (4\% in series of Baek et al) ${ }^{11}$ and PCD (12\% in the series of Lambiase et al). ${ }^{29}$ However, in our study and some recent studies (Rajak et al 1998,33 Yu et al 2004),36 both the procedures were found to be safe if performed properly with minimal complications. There was no mortality in either of the study groups.

One limitation of our study is that the aetiology of abscess was not uniform and formed a heterogeneous group with abscesses of both amoebic and pyogenic aetiology existing in both groups. Also, about $12 \%$ of the abscesses were of indeterminate aetiology. Anaerobic culture was not performed and no studies to detect fungus were done.

\section{CONCLUSION}

With the advancements in imaging techniques, ultrasound guided percutaneous treatment (aspiration or catheter drainage) has replaced open surgical intervention in the treatment of liver abscess, both procedures are safe with minimal complications. Percutaneous catheter drainage is a better modality as compared to percutaneous needle aspiration. Significant earlier clinical improvement $(p=0.039)$ and less time for $50 \%$ reduction in abscess cavity $(\mathrm{p}=0.000)$ and significant reduction of total or near total abscess cavity $(p=0.000)$ in the percutaneous catheter drainage group. The chances of failure of percutaneous needle aspiration increased with the increase in size of abscess cavity to be aspirated $(p=0.006)$. There was no significant advantage of catheter drainage over needle aspiration in terms of duration of hospital stay and time needed.

\section{REFERENCES}

[1] Adams F. The Genuine Works of Hippocrates. Transl [from the Greek with a preliminary discourse and annotations] Vol. 1 \& Vol. 2. New York: William Wood \& Co., 1886: p. 57, 58, 266, 267.

[2] Thompson JE Jr, Forlenza S, Verma R. Amoebic liver abscess: a therapeutic approach. Rev Infect Dis 1985;7(2):171-9.
[3] Pitt HA. Surgical management of hepatic abscesses. World J Surg 1990;14(4):498-504.

[4] Sherlock S, Dooley J. Diseases of the liver and billiary system. $9^{\text {th }}$ edn. Oxford: Blackwell Science Publications 1993: p. 471-502.

[5] Theron P. Surgical aspects of amoebiasis. Br Med J 1947;2(4516):123-6.

[6] Satiani B, Davidson ED. Hepatic abscesses: improvement in mortality with early diagnosis and treatment. Am J Surg 1978;135(5):647-50.

[7] Gerzof SG, Johnson WC, Robbins AH, et al. Intrahepatic pyogenic abscesses: treatment by percutaneous drainage. Am J Surg 1985;149(4):487-94.

[8] Attar B, Levendoglu H, Cuasay NS. CT-guided percutaneous aspiration and catheter drainage of pyogenic liver abscesses. Am J Gastroenterol 1986;81(7):550-5.

[9] Seeto RK, Rockey DC. Pyogenic liver abscess. Changes in etiology, management and outcome. Medicine (Baltimore) 1996;75(2):99-113.

[10] Singh JP, Kashyap A. A comparative evaluation of percutaneous catheter drainage for resistant amoebic liver abscesses. Am J Surg 1989;158(1):58-62.

[11] Baek SY, Lee MG, Cho KS, et al. Therapeutic percutaneous aspiration of hepatic abscesses: effectiveness in 25 patients. Am J Roentgenol 1993;160(4):799-802.

[12] Giorgio A, Tarantino L, Mariniello N, et al. Pyogenic liver abscesses: 13 years of experience in percutaneous needle aspiration with US guidance. Radiology 1995;195(1):122-4.

[13] Urbaniak GC, Plous S. Research randomizer (version 3.0) [Retrieved on November 7, 2011]. http://www.randomizer.org.

[14] Cook GC. Gastroenterological emergencies in the tropics. Bailliere's Clin Gastroenterol 1991;5(4):86186.

[15] Reeder MM. Tropical diseases of the liver and bile ducts. Semin Roentgenol 1975;10(3):229-43.

[16] Hughes MA, Petri WA Jr. Amebic liver abscess. Infect Dis Clin North Am 2000;14(3):565-82, viii.

[17] Chiu CT, Lin DY, Wu CS, et al. A clinical study on pyogenic liver abscess. Taiwan Yi Xue Hui Za Zhi 1987;86(4):405-12.

[18] Barnes PF, De Cock KM, Reynolds TN, et al. A comparison of amoebic and pyogenic abscess of the liver. Medicine (Baltimore) 1987;66(6):472-83.

[19] Hoffner RJ, Kilaghbian T, Esekogwu VI, et al. Common presentations of amoebic liver abscess. Ann Emerg Med 1999;34(3):351-5.

[20] Branum GD, Tyson GS, Branum MA, et al. Hepatic abscess. Changes in etiology, diagnosis and management. Ann Surg 1990;212(6):655-62.

[21] Sharma MP, Kumar A. Liver abscess in children. Indian J Pediatr 2006;73(9):813-7.

[22] Khan R, Hamid S, Abid S, et al. Predictive factors for early aspiration in liver abscess. World J Gastroenterol 2008;14(13):2089-93.

[23] Patterson M, Healy GR, Shabot JM. Serologic testing for amoebiasis. Gastroenterology 1980;78(1):136-41. 
[24] Pérez AJA, González JJ, Baldonedo RF, et al. Clinical course, treatment and multivariate analysis of risk factors for pyogenic liver abscess. Am J Surg 2001;181(2):177-86.

[25] Chou FF, Sheen-Chen SM, Chen YS, et al. Single and multiple pyogenic liver abscesses: clinical course, etiology and results of treatment. World J Surg 1997;21(4):384-8, discussion 388-9.

[26] Wang JH, Liu YC, Lee SS, et al. Primary liver abscess due to Klebsiella pneumoniae in Taiwan. Clin Infect Dis 1998;26(6):1434-8.

[27] Donovan AJ, Yellin AE, Ralls PW. Hepatic abscess. World J Surg 1991;15(2):162-9.

[28] Stain SC, Yellin AE, Donovan AJ, et al. Pyogenic liver abscess. Modern treatment. Arch Surg 1991;126(8):991-6.

[29] Do H, Lambiase RE, Deyoe L, et al. Percutaneous drainage of hepatic abscesses: comparison of results in abscesses with and without intrahepatic biliary communication. Am J Roentgenol 1991;157(6):120912.

[30] Huang CJ, Pitt HA, Lipsett PA, et al. Pyogenic hepatic abscess. Changing trends over 42 years. Ann Surg 1996;223(5):600-9.
[31] Saraswat VA, Agarwal DK, Baijal SS, et al. Percutaneous catheter drainage of amoebic liver abscess. Clin Radiol 1992;45(3):187-9.

[32] Agarwal DK, Baijal SS, Roy S, et al. Percutaneous catheter drainage of amoebic liver abscesses with and without intra hepatic biliary communication: a comparative study. Eur J Radiol 1995;20(1):61-4.

[33] Rajak CL, Gupta S, Jain S, et al. Percutaneous treatment of liver abscesses: needle aspiration versus catheter drainage. Am J Roentgenol 1998;170(4):1035-9.

[34] Dietrick RB. Experience with liver abscess. Am J Surg 1984;147(2):288-91.

[35] Gupta SS, Singh O, Sabharwal G, et al. Catheter drainage versus needle aspiration in management of large ( $>10 \mathrm{~cm}$ diameter) amoebic liver abscesses. ANZ J Surg 2011;81(7-8):547-51.

[36] Yu SCH, Ho SS, Lau WY, et al. Treatment of pyogenic liver abscess: prospective randomized comparison of catheter drainage and needle aspiration. Hepatology 2004;39(4):932-8. 